\title{
Photometric and spectroscopic investigation of 2867 Steins, target of the Rosetta mission ${ }^{\star}$
}

\section{Ground-based results prior to the Rosetta fly-by}

\author{
E. Dotto ${ }^{1}$, D. Perna ${ }^{1,2,3}$, S. Fornasier ${ }^{2,4}$, I. N. Belskaya ${ }^{5}$, M. A. Barucci ${ }^{2}$, V. G. Shevchenko ${ }^{5}$, \\ Yu. N. Krugly ${ }^{5}$, N. M. Gaftonyuk ${ }^{6}$, I. A. Tereschenko ${ }^{5}$, F. Scipioni ${ }^{1,3}$, and F. De Luise ${ }^{1}$ \\ ${ }^{1}$ INAF, Osservatorio Astronomico di Roma, via Frascati 33, 00040 Monteporzio Catone (Roma), Italy \\ e-mail: dotto@mporzio.astro.it \\ 2 LESIA, Observatoire de Paris, France \\ Università di Tor Vergata, Roma, Italy \\ ${ }^{4}$ Université de Paris 7 Denis Diderot, France \\ 5 Institute of Astronomy of Kharkiv Karazin National University, Kharkiv, Ukraine \\ ${ }^{6}$ Crimean Astrophysical Observatory, Crimea, Simeiz, Ukraine
}

Received 13 November 2008 / Accepted 18 December 2008

\section{ABSTRACT}

\begin{abstract}
Context. Rosetta is the cornerstone mission of ESA devoted to the study of minor bodies of the Solar System. During its journey to the comet 67P Churyumov-Gerasimenko, the main target, Rosetta also investigates two main belt asteroids, 2867 Steins (fly-by in September 2008) and 21 Lutetia (fly-by in July 2010).

Aims. In Spring 2008, we performed a broad observational campaign in order to complete the ground-based photometric and spectroscopic investigation of Steins. Before the Rosetta fly-by, this was the last opportunity to perform ground-based observations useful for calibrating properly the imaging and spectroscopic data obtained by the instruments on board the Rosetta spacecraft.

Methods. Visible photometry was carried out at a wide range of phase angles, and visible spectra were acquired at different rotational phases to retrieve information about the absolute magnitude and surface properties.

Results. The lightcurve was completely sampled in $V$ and $R$ bands. A rotational period of $6.057 \pm 0.003 \mathrm{~h}$ and color index $V-R=0.51 \pm 0.03$ mag were computed. We investigate Steins' phase relation over the range between 3.3 and 42 degrees in solar phase angle. The opposition effect is not evident down to the phase angle of 3 degrees, as is typical of other E-type asteroids. Assuming for Steins an opposition surge similar to that of other E-type asteroids, we calculated an absolute magnitude $H_{R}=12.81 \pm 0.03$, and slope parameter $G_{R}=0.42 \pm 0.02$. Eight visible spectra, obtained at different rotational phases, exhibit similar behavior, confirming a homogeneous composition of the asteroid surface and the $\mathrm{E}[\mathrm{II}]$ classification. All spectra display a spectral feature centered on about $0.49 \mu \mathrm{m}$ that is typical of E-type asteroids and usually attributed to the presence of sulfides (e.g. oldhamite). A tentative model of the surface composition is presented.
\end{abstract}

Key words. techniques: photometric - minor planets, asteroids - solar system: general

\section{Introduction}

The ESA Rosetta spacecraft was launched in March 2004. During its journey to its main target, the comet 67P Churyumov-Gerasimenko, Rosetta also performs the fly-by of two main belt asteroids: 2867 Steins, visited in September 2008, and 21 Lutetia, whose fly-by is scheduled for July 2010.

Steins is a small object, measuring a few $\mathrm{km}$ in diameter. Ground-based observations of this body were initiated only in January 2004, when it was first included in the list of possible Rosetta asteroid targets, and later selected with 21 Lutetia. In March 2004, Steins was observed photometrically by both Warner (2004) and Hicks et al. (2004), who measured synodic periods of $6.05 \pm 0.01 \mathrm{~h}$ and $6.06 \pm 0.05 \mathrm{~h}$, respectively. A full analysis of the latter set of observations was reported by Weissman et al. (2007), which obtained a revised period of $6.048 \pm 0.007 \mathrm{~h}$. Imaging performed by the Narrow

* Based on observations carried out at the Telescopio Nazionale Galileo, La Palma, Spain (AOT17/08A Programme TAC_28).
Angle Camera (NAC) of the Optical Spectroscopic and Infrared Remote Imaging System (OSIRIS) during the cruise phase of the Rosetta spacecraft, allowed Kuppers et al. (2007) and Jorda et al. (2008) to derive $P_{\text {syn }}=6.052 \pm 0.007 \mathrm{~h}$ and $P_{\text {syn }}=$ $6.054 \pm 0.003 \mathrm{~h}$, respectively. By compiling a set of 26 visible lightcurves belonging to 6 data sets obtained by ground-based observatories and one by the spacecraft, Lamy et al. (2008a) computed a sidereal period of $6.04681 \pm 0.00002 \mathrm{~h}$.

Visible and near-infrared spectroscopic observations of Steins obtained by Barucci et al. (2005) revealed a spectral behavior similar to that of E-type asteroids, with the presence of a strong feature at about $0.5 \mu \mathrm{m}$, a weaker feature at about $0.96 \mu \mathrm{m}$, and a flat and featureless behavior beyond $1 \mu \mathrm{m}$. On the basis of these results, Steins appears to be a quite evolved body that probably experienced high heating episodes.

Steins' albedo was derived by Fornasier et al. (2006) on the basis of polarimetric observations carried out at ESO-VLT during the summer of 2005. Using the empirical relationship with the polarimetric slope, Fornasier et al. (2006) determined an 
Table 1. Observational circumstances.

\begin{tabular}{lccccc}
\hline \hline Date & $\begin{array}{c}\Delta \\
(\mathrm{AU})\end{array}$ & $\begin{array}{c}\Delta \\
(\mathrm{AU})\end{array}$ & $\begin{array}{c}\alpha \\
(\mathrm{deg})\end{array}$ & filter & Observatory \\
\hline 31 Mar. 08 & 2.387 & 1.442 & 10.05 & $\mathrm{Vr}$ & Kharkiv \\
10 Apr. 08 & 2.370 & 1.385 & 5.77 & $\mathrm{vr}$ & Kharkiv \\
12 Apr. 08 & 2.368 & 1.378 & 5.03 & $\mathrm{VR}$ & Kharkiv \\
17 Apr. 08 & 2.359 & 1.361 & 3.50 & $\mathrm{VR}$ & TNG \\
18 Apr. 08 & 2.358 & 1.359 & 3.36 & VR & TNG \\
19 Apr. 08 & 2.356 & 1.357 & 3.29 & VR & TNG \\
20 Apr. 08 & 2.354 & 1.355 & 3.30 & VR & TNG \\
02 May 08 & 2.335 & 1.353 & 7.34 & VR & Kharkiv \\
04 May 08 & 2.331 & 1.356 & 8.27 & BVR & Kharkiv \\
10 May 08 & 2.322 & 1.372 & 11.01 & VR & Kharkiv \\
11 May 08 & 2.320 & 1.375 & 11.46 & VR & Kharkiv \\
31 May 08 & 2.287 & 1.485 & 19.38 & VR & Simeiz \\
02 Jun. 08 & 2.284 & 1.499 & 20.01 & VR & Kharkiv \\
03 Jun. 08 & 2.282 & 1.507 & 20.33 & VR & Kharkiv \\
\hline
\end{tabular}

albedo of $0.45 \pm 0.10$. This high value is consistent with the Etype classification suggested by the spectroscopic behavior. A new determination of the geometric albedo, obtained by thermal modeling of Spitzer data, inferred a value $p_{V}(H, G)=0.34 \pm 0.06$ (Lamy et al. 2008b). This value appears to be low for an E-type object, although the Spitzer emissivity spectrum between 5.2 and $38 \mu \mathrm{m}$ is consistent with that of aubrite meteorites and enstatite minerals (Barucci et al. 2008), and confirms the E-type classification.

The radiometric method, of course, requires both thermal data and visual observations, and input values such as the asteroid absolute magnitude $H$ and the phase slope parameter $G$. To derive these parameters with sufficient accuracy, the phase relation, that is the reduced visual magnitude (at $1 \mathrm{AU}$ of geocentric and heliocentric distances) of the asteroid as a function of phase angle, must be well sampled, particularly at small phase angles.

Until April 2008, neither photometric observations nor lightcurves of Steins were available for phase angles $\alpha<7^{\circ}$. To investigate and assess the physical properties of Steins before the Rosetta encounter, we carried out low phase angle visible spectroscopy, and visible photometry of this asteroid over a wide range of phase angles, between 31st March and 3rd June 2008. These observations are important to complete the groundbased data set, and to compute the physical parameters of Steins that are fundamental for calibrating properly the imaging and spectroscopic data acquired by instruments onboard the Rosetta spacecraft.

\section{Observations and data reduction}

Observations were carried out at the Institute of Astronomy of the Kharkiv Karazin National University $(70 \mathrm{~cm}$ reflector), the Simeiz Department of the Crimean Astrophysical Observatory ( $1 \mathrm{~m}$ reflector), and the Telescopio Nazionale Galileo (TNG, 3.6 m La Palma, Spain) (see Table 1).

During the photometric observing runs, we also acquired bias, flat-field, and calibration images. Several standard stars covering a range of stellar types (Landolt 1992) were observed for a wide range of airmasses. The CCD images were reduced and calibrated in the standard way (see e.g. Dotto et al. 2006). First, bias and flat-field corrections were performed: a master flat field was created by obtaining the median of several flat fields acquired during twilight. Then the instrumental magnitudes were computed, with the zero point, extinction, and color terms necessary to convert these instrumental magnitudes into apparent
Table 2. Visible spectroscopy performed at TNG.

\begin{tabular}{cccccccc}
\hline \hline$\#$ & Date & $\begin{array}{c}\text { UT } \\
\text { (start) } \\
\text { (h:m) }\end{array}$ & Texp & airm & $\begin{array}{c}\text { Sol } \\
\text { an }\end{array}$ & $\begin{array}{c}\text { airm } \\
\text { sol } \\
\text { an }\end{array}$ & $\begin{array}{c}\text { Spect. } \\
\text { slope } \\
\text { S(\%10 } \%^{3} \text { A) }\end{array}$ \\
\hline 1 & 17 Apr. & $23: 56$ & 900 & 1.27 & 1 & 1.16 & $6.88 \pm 0.65$ \\
2 & 18 Apr. & $23: 56$ & 900 & 1.25 & 2 & 1.14 & $7.09 \pm 0.65$ \\
3 & 19 Apr. & $03: 04$ & 900 & 1.36 & 2 & 1.14 & $7.12 \pm 0.65$ \\
4 & 20 Apr. & $00: 03$ & 600 & 1.23 & 1 & 1.19 & $7.48 \pm 0.65$ \\
5 & 20 Apr. & $00: 15$ & 900 & 1.22 & 1 & 1.19 & $8.26 \pm 0.65$ \\
6 & 20 Ap.r & $00: 34$ & 900 & 1.20 & 1 & 1.19 & $7.75 \pm 0.65$ \\
7 & 20 Apr. & $01: 29$ & 900 & 1.19 & 1 & 1.19 & $7.25 \pm 0.65$ \\
8 & 20 Apr. & $01: 50$ & 900 & 1.21 & 1 & 1.19 & $6.48 \pm 0.65$ \\
\hline
\end{tabular}

1. La 102-1081; 2. hd 144873 .

magnitudes. The calibrated data were corrected for lightime. The error bars take into account both the instrumental errors, given by statistics alone, and the calibration errors.

Visible spectroscopy was acquired at the TNG telescope, using DOLORES equipped with the low resolution blue (LR-B) and red (LR-R) grisms. The observational circumstances are reported in Table 2. Spectra were acquired through a 2 arcsec wide slit oriented along the parallactic angle to avoid flux loss due to the differential refraction. During the observing run, we also acquired bias, flat field, calibration lamp (He lines for grism LR-B and Ne-Ar lines for grism LR-R), and spectra of several solar analog stars. The spectroscopic data reduction was completed using MIDAS and IDL and by applying standard procedures (see e.g. Dotto et al. 2006). The reflectivity of Steins was then obtained by dividing its spectrum by that of the solar analog star closest in time and airmass to the object, as reported in Table 2.

\section{Results}

\subsection{Composite lightcurves}

The rotational synodic period was computed by applying the method based on Fourier analysis of lightcurves, developed by Harris et al. (1989a). A value of $P_{\text {syn }}=6.057 \pm 0.003 \mathrm{~h}$ was obtained.

Figure 1 shows the composite lightcurves derived for the $V$ and $R$ bands. The measured lightcurves in the $V$ and $R$ bands are consistent within the error bars and characterized by rather symmetrical shape. The lightcurve amplitude at minimum phase angle is $0.20 \pm 0.02 \mathrm{mag}$ in both bands, which provides a lower limit to the semimajor axis ratio $a / b \geq 1.20 \pm 0.02$, assuming that Steins does not have considerable albedo variations and that the observed amplitude is only affected by the asteroid shape. A slight increase in amplitude toward larger phase angles is seen in our observations.

The composite lightcurves were used to convert all measured magnitudes into those of the same portion of the surface, corresponding to the lightcurve maximum, which is known to be less affected by asteroid shape. Since most of our observations covered one or both maxima, we can reconstruct the magnitudephase dependence of Steins to good accuracy. To derive mean magnitudes, we added half of the lightcurve amplitude to each magnitude in the lightcurve maximum. The calculated mean magnitudes in the $R$ and $V$ bands are reported in Table 3. The resulting $V-R$ color is $0.51 \pm 0.03 \mathrm{mag}$.

\subsection{Magnitude-phase dependence}

The magnitude-phase dependence in $R$ band is shown in Fig. 2 . We also used previously published observations obtained in 


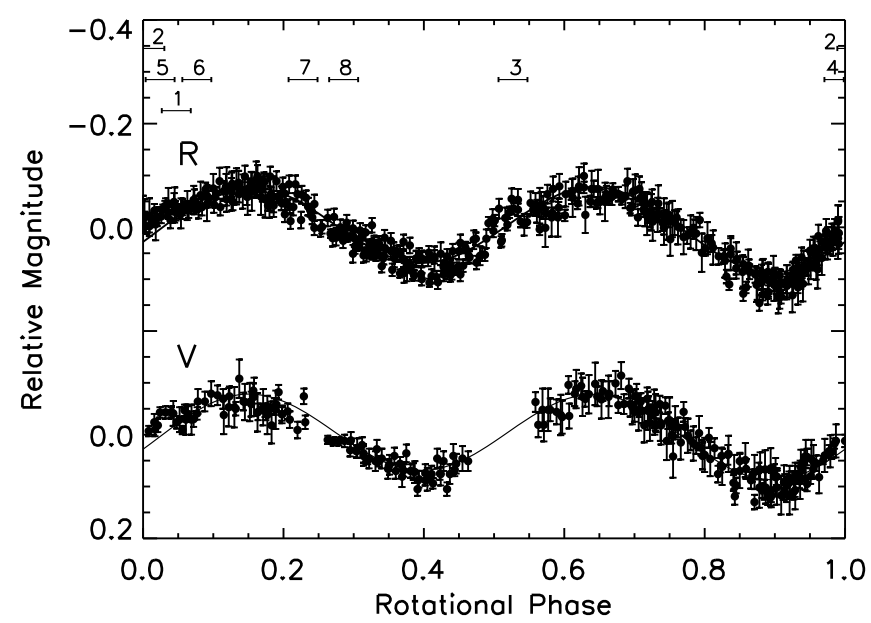

Fig. 1. Composite $R$ and $V$ lightcurves based on our observations performed in March-June 2008. The coverage of the obtained spectra is shown on the top of the data. The lightcurve zero point is at $0 \mathrm{UT}$ on 2008 April 19th.

Table 3. $V$ and $R$ magnitudes.

\begin{tabular}{lccc}
\hline \hline Date & $\begin{array}{c}\alpha \\
(\mathrm{deg})\end{array}$ & $\begin{array}{c}R \\
(\mathrm{mag})\end{array}$ & $\begin{array}{c}V \\
(\mathrm{mag})\end{array}$ \\
\hline 12 Apr. 08 & 5.03 & $13.14 \pm 0.02$ & $13.67 \pm 0.02$ \\
17 Apr. 08 & 3.50 & $13.13 \pm 0.02$ & $13.65 \pm 0.02$ \\
18 Apr. 08 & 3.36 & $13.11 \pm 0.03$ & $13.64 \pm 0.03$ \\
19 Apr. 08 & 3.29 & $13.12 \pm 0.02$ & $13.64 \pm 0.02$ \\
20 Apr. 08 & 3.30 & $13.12 \pm 0.04$ & $13.66 \pm 0.04$ \\
02 May 08 & 7.34 & $13.22 \pm 0.02$ & $13.72 \pm 0.02$ \\
04 May 08 & 8.27 & $13.26 \pm 0.02$ & $13.75 \pm 0.02$ \\
10 May 08 & 11.01 & $13.33 \pm 0.03$ & $13.81 \pm 0.03$ \\
11 May 08 & 11.46 & $13.35 \pm 0.02$ & $13.83 \pm 0.02$ \\
31 May 08 & 19.38 & $13.55 \pm 0.02$ & - \\
02 Jun. 08 & 20.01 & $13.52 \pm 0.03$ & $14.02 \pm 0.03$ \\
03 Jun. 08 & 20.33 & $13.53 \pm 0.02$ & $14.04 \pm 0.03$ \\
\hline
\end{tabular}

2004-2008 by Hicks et al. (2004), Weissman et al. (2007), Jorda et al. (2008), and Lamy et al. (2008a). These measurements were corrected for the lightcurve amplitude in the same way as our observations acquired in 2008. One can see the good agreement between the data obtained at different oppositions. It confirms that the rotation axis of Steins is almost perpendicular to the ecliptic plane (Lamy et al. 2008a) and thus that Steins is observed at almost the same aspect angle in different apparitions (aspect angle $\xi \sim 86^{\circ}$ ). All of these data were used to compute the absolute magnitude $H$ and slope parameter $G$. According to the formalism of Bowell et al. (1989), we obtained $H_{R}=12.86 \pm 0.02$ and $G_{R}=0.42 \pm 0.02$. Our $G_{R}$ value is higher than that obtained in previous papers. This discrepancy could be due to a lack of observations at phase angle lower than $7^{\circ}$ in previously analyzed samples. The magnitude-phase dependence is consistent with the linear behavior for the observed range of phase angles with $\beta_{R}=0.026 \pm 0.001$ and $R(1,0)=13.04 \pm 0.02$ (see Fig. 2). The shallow phase slope observed for Steins strongly supports the E-type classification of its surface.

A comparison of the phase-dependence of Steins and other E-type asteroids is shown in Fig. 3. All of these data were obtained in the $V$-band and shifted in magnitude to match the Steins data.

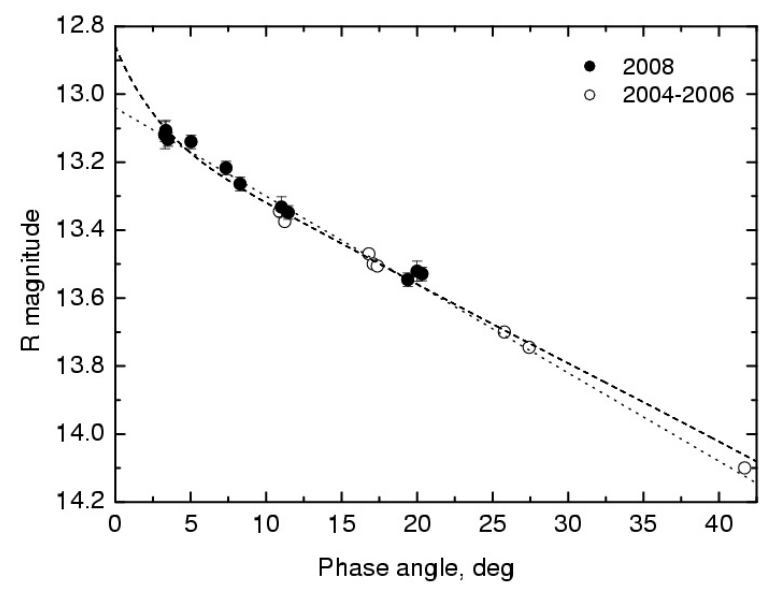

Fig. 2. Phase curve of Steins in the $R$ band fitted by linear (dotted line) and HG fit (dashed line).

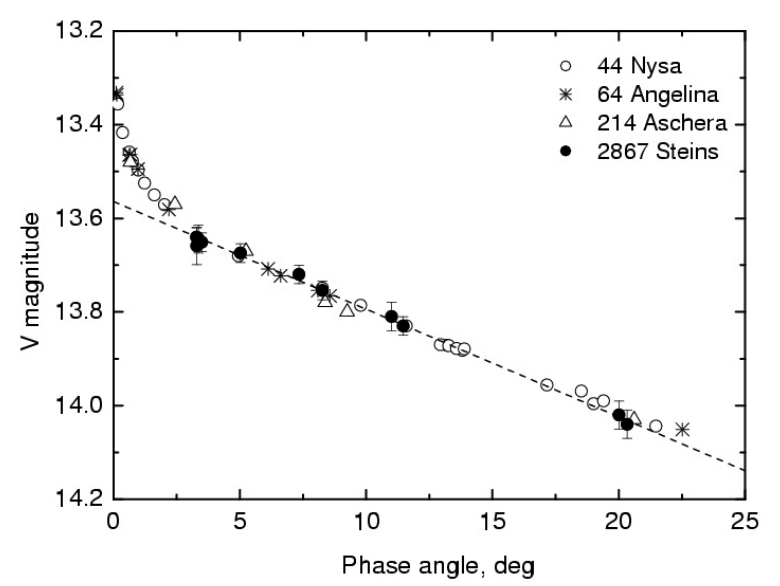

Fig. 3. Magnitude-phase dependence in the $V$ band of Steins and other E-type asteroids from Harris et al. (1989b) and Belskaya et al. (2003).

By assuming that the amplitude of the opposition effect of Steins is similar to that of the other E-type asteroids $(0.23 \pm 0.03$ by Belskaya \& Shevchenko 2000), we can calculate the Steins' $R$ absolute magnitude by adding the value of the opposition effect to $R(1,0)$. We obtain $H_{R}=12.81 \pm 0.03$.

\subsection{Spectral properties and modeling}

During the TNG observing run in April 2008, we also obtained the eight visible spectra shown in Fig. 4. Their locations within the Steins lightcurve are indicated in the top of Fig. 1. All the obtained spectra exhibit the same behavior that is typical of Etype asteroids, in particular of the E[II] subgroup, as identified by Gaffey \& Kelley (2004). The spectrum is almost flat and featureless between $0.55-0.9 \mu \mathrm{m}$, and shows an absorption signature centered on about $0.49 \mu \mathrm{m}$, typical of E-type objects and usually attributed to sulfides such as troilite (Cloutis \& Burbine 1999) and oldhamite (see e.g. Fornasier et al. 2008, and references therein). The spectral slopes computed between 0.56 and $0.79 \mu \mathrm{m}$ are reported in Table 2. Since we find the same spectral behavior over $30 \%$ of the rotational lightcurve, we can argue that Steins has a quite homogeneous surface composition.

In Fig. 5, the blue grism spectrum \#2 and the red grism spectrum \#5, which cover the same surface region, are plotted together. To investigate the surface composition of Steins, we attempt to reproduce its spectral behavior using geographical 


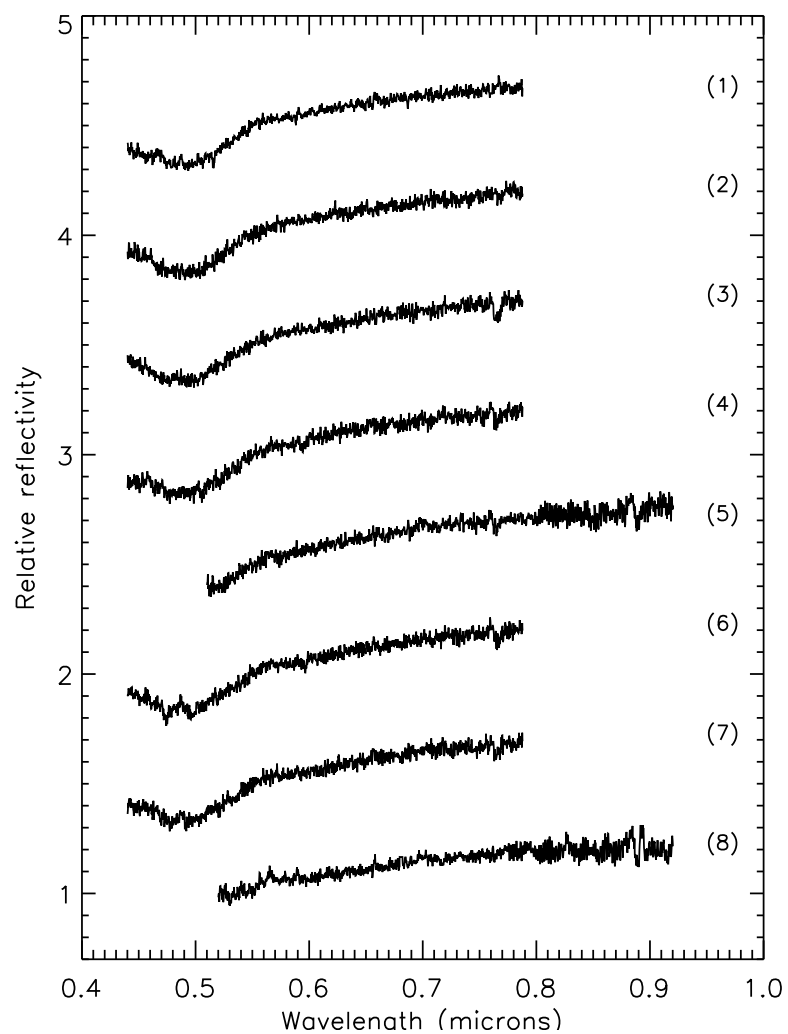

Fig. 4. Visible spectra of Steins normalized at $0.55 \mu \mathrm{m}$ and shifted by 0.5 for clarity. The numbers on the right correspond to the spectrum numbers reported in Table 2 and Fig. 1.

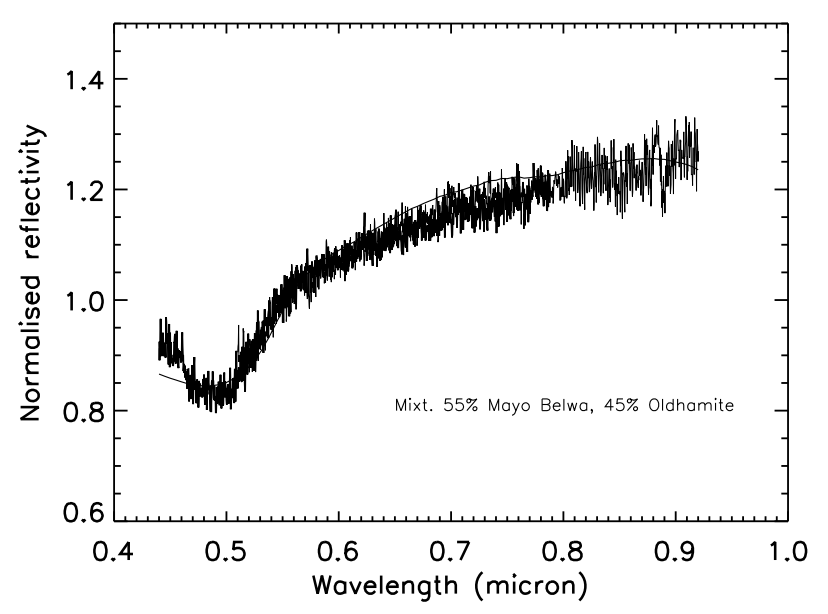

Fig. 5. Spectra (\#2) and (\#5) normalized at $0.55 \mu \mathrm{m}$. The continuous line shows the tentative model of the Steins surface composition.

mixtures of different components selected from enstatite meteorites and correlated minerals included in the RELAB catalog. We ran a radiative transfer model, based on the Hapke theory, that had already been used to model the surface composition of Jupiter Trojans, Trans-Neptunian Objects, and Centaurs (see e.g. Dotto et al. 2003, 2006). The continuous line in Fig. 5 shows the synthetic spectrum of a mixture consisting of $55 \%$ enstatite achondrite meteorite (aubrite) Mayo Belwa and $45 \%$ oldhamite. This mixture has an albedo of 0.35 , in good agreement with the albedo of Steins derived from Spitzer data (Lamy et al. 2008b).

On the basis of these results, the surface of Steins appears to be homogeneous and its composition, as already suggested by both Barucci et al. (2005) and Fornasier et al. (2007, 2008), resembles the enstatite meteorites, in particular the aubrites, enriched in oldhamite.

\section{Conclusions}

A broad observational campaign of Steins was carried out between 31st March and 3rd June 2008. Low phase angle visible spectroscopy and visible photometry at a wide range of phase angles were performed for this main belt asteroid.

On the basis of these observations, we have derived:

1. $V$ and $R$ lightcurves, which allowed us to compute a synodical rotational period of $6.057 \pm 0.003 \mathrm{~h}$ and a $V-R$ color index of $0.51 \pm 0.03 \mathrm{mag}$.

2. the magnitude-phase dependence. We computed a slope parameter of $G_{R}=0.42 \pm 0.02$ and absolute magnitude of $H_{R}=12.81 \pm 0.03$.

3. eight visible spectra acquired at different rotational phases. All of the spectra exhibit an absorption feature centered on about $0.49 \mu \mathrm{m}$, which is usually attributed to sulfides. Since all of the acquired visible spectra, covering about $30 \%$ of the rotational lightcurve, are similar to each other, we have argued that the surface of Steins is quite homogeneous. From the observed spectral behavior, we confirmed the classification of Steins as an E-type asteroid and that its surface composition is similar to enstatite achondrite meteorites enriched in oldhamite.

Based on our derived value of absolute magnitude, Steins appears to be fainter than predicted by previous observations. Its photometric properties, mainly the low slope of the phase relation, and its spectral behavior provide conclusive proof of its E-type classification.

These results constitute the last ground-based data set for Steins acquired prior to the Rosetta fly-by. These data are useful in the assessment of the physical nature of Steins, and will provide a fundamental basis for the calibration, analysis, and interpretation of imaging and spectroscopic data acquired by the instruments OSIRIS and VIRTIS onboard the Rosetta spacecraft.

Acknowledgements. We thank the reviewers, A.W. Harris and S.J. Bus, whose suggestions improved the manuscript.

\section{References}

Barucci, M. A., Fulchignoni, M., Fornasier, S., et al. 2005, A\&A, 430, 313 Barucci, M. A., Fornasier, S., Dotto, E., et al., 2008, A\&A, 477, 665 Belskaya, I. N., \& Shevchenko, V. G. 2000, Icarus, 147, 94 Belskaya, I. N., Shevchenko, V. G., Kiselev, N. N., et al. 2003, Icarus, 166, 276 Bowell, E., Hapke, B., Domingue, D., et al. 1989, in Asteroids II (Univ. of Arizona Press), 524

Cloutis, E. A., \& Burbine, T. H. 1999, LPS Conference XXX, abstract No. 1875 Dotto, E., Barucci, M. A., Boehnhardt, H., et al. 2003, Icarus, 162, 408 Dotto, E., Fornasier, S., Barucci, M. A., et al. 2006, Icarus, 183, 420 Fornasier, S., Belskaya, I., Fulchignoni, M., et al. 2006, A\&A, 449, L9 Fornasier, S., Marzari, F., Dotto, E., et al. 2007, A\&A, 474, 29 Fornasier, S., Migliorini, A., Dotto, E., et al. 2008, Icarus, 196, 119 Gaffey, M. J., \& Kelley, M. S. 2004, LPS Conference, abstract No. 1812 Harris, A. W., Young, J., Bowell, E., et al. 1989a, Icarus, 77, 171 Harris, A. W., Young, J. W., Contreiras, L., et al. 1989b, Icarus, 81, 365 Hicks, M. D., Bauer, \& Tokunaga, A. T. 2004, IAU Circ., 8315 Jorda, L., Lamy, P. L., Faury, G., et al. 2008, A\&A, 487, 1171 Kuppers, M., Mottola, S., Lowry, S. C., et al. 2007, A\&A, 462, L13. Lamy, P. L., Kaasalainen, M., Lowry, S. C., et al. 2008a, A\&A, 487, 1179 Lamy, P. L., Jorda, L., Fornasier, S., et al. 2008b, A\&A, 487, 1187 Landolt, A. U. 1992, AJ, 104, 340

Warner, B. D. 2004, Minor Planet Bul., 31, 67

Weissman, P. R., Lowry, S. C., \& Choi, Y. L. 2007, A\&A, 466, 737 